\title{
Editorial
}

\section{Greetings from the New Chief Editor}

\author{
Hiroki Sayama $\mathbb{D}^{1,2}$ \\ ${ }^{1}$ Center for Collective Dynamics of Complex Systems, \\ Department of Systems Science and Industrial Engineering Binghamton University, State University of New York, \\ New York, USA \\ ${ }^{2}$ School of Commerce, Waseda University, Tokyo, Japan
}

Correspondence should be addressed to Hiroki Sayama; complexity@hindawi.com

Received 13 March 2020; Accepted 17 March 2020; Published 2 June 2020 Copyright (c) 2020 Hiroki Sayama. This is an open access article distributed under the Creative Commons Attribution License, which
permits unrestricted use, distribution, and reproduction in any medium, provided the original work is properly cited.

Complexity is pleased to announce the installment of Prof Hiroki Sayama as its new Chief Editor. In this Editorial, Prof Sayama describes his feelings about his recent appointment, discusses some of the journal's journey and relevance to current issues, and shares his vision and aspirations for its future.

As of June 2019, I have been appointed as the new Chief Editor of Complexity, an internationally recognized signature journal of complexity science. The relevance of complexity science to diverse, real-world, complex problems has been increasingly recognized and acknowledged over the last few decades across a wide range of scientific domains and applications. This is especially true this year, as we are all aware, due to the global pandemic of COVID-19 which has made everyone realize how important yet counter-intuitive some of the aspects of complex dynamical systems are, including exponential growth, time delay, contact networks, human mobility, percolation, and phase transition. As one of the leading open access journals of the field, Complexity's mission to swiftly disseminate state-of-the-art discoveries in complexity science is more critical than ever. I will do my very best to work toward this goal as the Chief Editor.

Complexity was launched in 1995 by John Wiley \& Sons and was flipped to an open access publishing model when Hindawi took over publishing the journal in 2017 as part of the Wiley-Hindawi partnership. Throughout its quarter-century history, this journal has published many innovative, influential, and highly impactful articles on the scientific study of complex systems. One can search for the journal on any citation database to glimpse the impact this journal has been making on complexity science. It is hence my greatest honor and pleasure to have an opportunity to serve for this established, highly reputed journal that has been vital to the field.
I must confess that I was initially surprised when Hindawi approached me about the Chief Editor role last year. This was first because all the earlier Editors-in-Chief and Executive Editors of this journal were highly influential, accomplished scientists, so I would have never imagined I could move into their shoes. But another reason for my surprise, to be honest, was that prior to my appointment I was frequently in touch with Hindawi with concerns and misgivings. As an Academic Editor for Complexity, I was already involved in its editorial and peer review processes and often felt that the editorial workflow was not clear enough and that the journal was drifting apart from the active community of complex systems researchers. These issues were largely due to the transition of the journal from one publisher to another-the system was (and still is) going through exploration and adaptation to a new environment and context.

For me, appointing such a critical person as the Chief Editor of a journal indicates something very open, constructive, and adaptive about Hindawi. I even told them my intention to become an Editor that serves the whole research community, not just their journal, and yet their enthusiasm was not deterred. They are seriously looking for ways to improve the journal, for which I am grateful.

There are several things I would like to achieve during my tenure as the Chief Editor.

First and foremost, I strive to establish a strong, transparent channel of communication and collaboration 
between the global community of complex systems researchers and the editorial team of the journal. Complexity has been serving-and will continue to serve-the complexity science community at large. Its editorial processes and practices should, therefore, incorporate what the community needs and expects. Through close communication and collaboration, I hope to make this journal home for and led by the global community of complex systems researchers. As part of this effort, we have recently formed a team of Associate Editors, whose names are now available on the journal's website.

Second, I will make sure the theme of the journal is clearly set on complexity science. This unique thematic focus is what makes Complexity valuable and attractive for authors and readers and distinct from other similar journals. Thanks to Carlos Gershenson and others, the journal website now has a clearly stated Aims and Scope description. I would encourage potential authors to carefully review them and clearly articulate in their cover letter what contribution their work makes to the field of complex systems. Meanwhile, this journal has a highly interdisciplinary audience, coming from many different backgrounds, and authors are reminded that they should make their papers broadly accessible to such an audience.

Third, I plan to promote publications of educational and pedagogical value, as well as work related to complex systems on societal outreach. This scientific field has reached a level of maturity and has begun producing real impacts on the next generation and society at large. A recent example of educational outreach is Complexity Explained [1], a collaboratively produced online educational resource and booklet led by Manlio De Domenico and others. Another example is Complexity Explorables [2] by Dirk Brockmann that offers many interactive demonstrations of canonical complex systems models (some were included in Complexity Explained too). Complexity welcomes contributions on such education and societal outreach activities, not just on scientific research.

Fourth, I would like to explore Open Access, and Open Science initiatives more broadly, to improve the dissemination of scientific discoveries. There are already many OA journals out there, but it is sometimes questionable whether the original vision and mission of the OA movement are still maintained. In this regard, Hindawi is fully committed to the core OA philosophy and values and is open to implement changes and explore best practices with us. Such a strong commitment to the OA philosophy and values is particularly crucial when science needs to meet societal demands at a time of uncertainty. We will all work together to seek better OA publication models that are accessible to both authors and readers, maximizing the values for the society at large, while remaining sustainable for publishers. Some improvements are already in the works, as demonstrated by Hindawi's recent initiatives in response to the COVID-19 outbreak [3].

That said, the future directions of the journal are not limited only to those listed above. I keep myself wide open to any suggestions and comments. One promise I make here is that I commit myself to serve as an honest channel that connects authors, readers, reviewers, editors, and publishers in an open, efficient, transparent conversation and collaboration. I ask for your support, contribution, and participation in helping Complexity continue to grow as the best place to discuss complexity science.

\section{References}

[1] M. De Domenico, D. Brockmann, C. Camargo et al., "Complexity explained," 2019, https://complexityexplained.github.io/.

[2] D. Brockmann, "Complexity explorables," 2019, https://www. complexity-explorables.org/.

[3] S. Greaves, "Sharing findings related to COVID-19 in these extraordinary times," 2020, https://www.hindawi.com/post/ sharing-findings-related-covid-19-these-extraordinary-times/. 